\title{
Assessment of mixture toxicity of (tri)azoles and their hepatotoxic effects in vitro by means of omics technologies
}

\author{
Bettina Seeger ${ }^{1}$ (1) $\cdot$ Almut Mentz $^{3} \cdot$ Constanze Knebel $^{2} \cdot$ Flavia Schmidt $^{2} \cdot$ Hanna Bednarz $^{3} \cdot$ Karsten Niehaus $^{3}$. \\ Stephan Albaum ${ }^{3} \cdot \mathrm{Jörn} \mathrm{Kalinowski}^{3} \cdot$ Thomas Noll $^{3} \cdot$ Pablo Steinberg $^{1,4} \cdot$ Philip Marx-Stoelting $^{2}$ (1) $\cdot$ Tanja Heise $^{2}$
}

Received: 26 April 2019 / Accepted: 17 June 2019 / Published online: 28 June 2019

(c) The Author(s) 2019

\begin{abstract}
Consumers are constantly exposed to chemical mixtures such as multiple residues of different pesticides via the diet. This raises questions concerning potential combination effects, especially because these substances are tested for regulatory purposes on an individual basis. With approximately 500 active substances approved as pesticides, there are too many possible combinations to be tested in standard animal experiments generally requested for regulatory purposes. Therefore, the development of in vitro tools and alternative testing strategies for the assessment of mixture effects is extremely important. As a first step in the development of such in vitro tools, we used (tri)azoles as model substances in a set of different cell lines derived from the primary target organ of these substances, the liver (human: HepaRG, rat: H4IIE). Concentrations were reconciled with measured tissue concentrations obtained from in vivo experiments to ensure comparable effect levels. The effects of the substances were subsequently analyzed by transcriptomics and metabolomics techniques and compared to data from corresponding in vivo studies. The results show that similar toxicity pathways are affected by substances and combinations, thus indicating a similar mode of action and additive effects. Two biomarkers obtained by the approach, CAR and Cyp1A1, were used for mixture toxicity modeling and confirmed the concentration-additive effects, thus supporting the selected testing strategy and raising hope for the development of in vitro methods suitable to detect combination effects and prioritize mixtures of concern for further testing.
\end{abstract}

Keywords Triazoles $\cdot$ Hepatotoxicity $\cdot$ mixture toxicology $\cdot$ Omics technologies $\cdot$ Risk assessment

Bettina Seeger, Almut Mentz, Constanze Knebel, Philip MarxStoelting and Tanja Heise contributed equally to this paper.

Electronic supplementary material The online version of this article (https://doi.org/10.1007/s00204-019-02502-w) contains supplementary material, which is available to authorized users.

Philip Marx-Stoelting

philip.marx-stoelting@bfr.bund.de

1 Institute for Food Toxicology and Center for Alternatives and Complementary Methods to Animal Experiments, University of Veterinary Medicine Hannover, Foundation, Hannover, Germany

2 Department for Pesticide Safety, German Federal Institute for Risk Assessment, Berlin, Germany

3 Center for Biotechnology, Bielefeld University, Bielefeld, Germany

4 Max Rubner-Institute, Federal Research Institute of Nutrition and Food, Karlsruhe, Germany

\section{Introduction}

Since consumers are constantly exposed to mixtures of chemicals, e.g., to multiple pesticide residues via the daily diet, it is important to take chemical mixtures into account in risk assessment. In the European Union (EU), the corresponding legislation such as Regulation (EC) No. 1107/2009 states that plant protection products (PPP) and their residues " [...] shall have no immediate or delayed harmful effect on human health, $[\ldots]$, taking into account known cumulative and synergistic effects where the scientific methods accepted by the Authority to assess such effects are available; [...]" (EC 2009). However, pesticide active substances are extensively tested on an individual basis in conventional standard toxicity tests, mostly in animal experiments, rather than in combination or as formulated products. These standardized animal studies facilitate the identification of target organs, toxic effects and no observed adverse effect levels (NOAELs) for the derivation of reference values for single 
active substances but are generally not subjected to a detailed mechanistic analysis. Such a mechanistic analysis is, however, relevant for the assessment of mixture toxicity, because the mode of action of substances in a mixture defines the nature of the potential combination effect (Kortenkamp et al. 2009; EFSA 2019; OECD 2018). Substances with a similar mode or mechanism of action (e.g., affecting the same target organ and toxicity pathway by interference with similar receptors) are considered to cause mixture effects in a doseor concentration-additive manner (DA/CA model, OECD 2018). Thus, there are two major challenges to mixture toxicity testing that may be solved by an alternative testing strategy making use of omics analysis and in vitro assays: the need for mechanistic understanding and the large number of possible combinations.

With approximately 500 active substances having been approved in Europe, there are far more possible binary or ternary combinations due to their occurrence as multiple residues (EFSA 2018) than one could test in animal studies for economical and ethical reasons (Solecki et al. 2014). Hence, the development of in vitro tools for the assessment of mixture effects and corresponding testing strategies are urgently needed.

The inclusion of data obtained using 'omics' techniques such as transcriptomics and metabolomics in the risk assessment of chemical mixtures' fuels hopes to uncover combined effects at a mechanistic level, giving hints for modes of action or adverse outcome pathways (AOPs) (Altenburger et al. 2012; Marx-Stoelting et al. 2015; Brockmeier et al. 2017). The AOP concept has become widely used in toxicology to link a molecular initiating event (MIE) as a first response of an organism to a chemical stressor at the molecular level via one or more key events (KEs) at the molecular and/or cellular level to an adverse outcome (AO) at the organism level (Ankley et al. 2010; Vinken 2013). AOPs may thus facilitate understanding mixture effects by helping to identify key toxicity pathways affected by substances and their combinations. Omics analysis may reveal AOPs and, consequently, MIEs such as the activation of nuclear receptors or other key signaling molecules that are involved. Results could then be used to differentiate between substances with a similar or a dissimilar mode of action and, thus, the nature of the mixture effect. Additionally, the comprehensive analysis of effects at a specific molecular target (e.g., the activation of a nuclear receptor) might further be used for modeling mixture effects. Therefore, in the present study a combined in vivo-in vitro approach was chosen making use of omics techniques to analyze mixture effects of azole fungicides at a mechanistic level in their major target organ.

Azole fungicides are a group of widely used fungicides designed to specifically inhibit a fungal CYP enzyme (CYP51) involved in membrane synthesis, which have been selected by EFSA as model compounds to build a cumulative assessment group, since exposure to multiple residues of azoles is likely to occur (EFSA 2009). The main target organ of azoles in mammals is the liver, but some of these substances also show developmental toxicity and effects on endocrine organs (EFSA 2009). Supplementary Table 1 summarizes the liver effects of the azole fungicides cyproconazole (C), epoxiconazole (E) and prochloraz (P) as observed in animal studies performed for regulatory toxicity testing. Cyproconazole (EFSA 2010), epoxiconazole (EFSA 2008), prochloraz (EFSA 2011) and their mixtures (Heise et al. 2018; Schmidt et al. 2016) are known to increase liver weight and lead to enzyme induction in rats and mice. In previous in vivo studies in rats, it was confirmed that the liver is a main target organ of azoles and that hepatotoxicity is induced by interference with the nuclear receptors constitutive androstane receptor (CAR), pregnane $\mathrm{X}$ receptor (PXR) and aryl hydrocarbon receptor (AhR) (Heise et al. 2015, 2018; Goetz and Dix 2009a, b). Therefore, a main aim of the present study was to determine if these events could be confirmed in cell lines of hepatic origin and if omics techniques would be able to identify corresponding toxicity pathways.

Further objectives of the present study were to identify appropriate markers or MIEs to model combination effects and to analyze if the assumption of EFSA (2009), assuming DA/CA, was correct or if synergistic or subadditive effects could be observed.

The above-mentioned azoles were tested in the human liver cell line HepaRG as well as in the rat liver cell line H4IIE to compare the in vitro data with data obtained in in vivo studies in rats (Heise et al. 2015, 2018; Schmidt et al. 2016) and to facilitate the extrapolation of results from rat to human. Effects of binary and ternary mixtures of the widely used triazoles cyproconazole, epoxiconazole and the imidazole prochloraz were investigated in vitro based on a multi-level-omics approach, thereby obtaining transcriptome and metabolome data.

\section{Materials and methods}

\section{Test substances}

Cyproconazole (CAS no. 94361-06-5, Batch no. CHF1E00042, purity 96.8\%) was obtained from Syngenta (Basel, Switzerland). Epoxiconazole (CAS no.133855-988, Batch no. 8563, purity 97.0\%) and prochloraz (CAS no. 67747-09-5, Batch no. COD-000718, purity 98.0\%) were supplied by BASF (Ludwigshafen, Germany). All test substances were dissolved in DMSO (CAS no.67-68-5, purity $\geq 99.5 \%$, Carl Roth, Karlsruhe, Germany) and stored at minus $20^{\circ} \mathrm{C}$. The compound concentrations in the cell 
culture medium tested to perform metabolome analyses are listed in Supplementary Table 2. The highest compound concentration was selected when wanting to perform transcriptome analyses. The maximal concentration of the solvent in the cell culture medium was $1 \%$. Cells were treated for $24 \mathrm{~h}$ with the single test substances [cyproconazole (C)/ epoxiconazole $(\mathrm{E}) /$ prochloraz $(\mathrm{P})]$, the binary $(\mathrm{CE})$ and the ternary mixtures (CEP) as well as with a solvent control in triplicates for metabolome analyses and six replicates for the transcriptome analyses. In a first step, different incubation times $(8 \mathrm{~h}, 24 \mathrm{~h}, 72 \mathrm{~h})$ were tested, with the $24 \mathrm{~h}$ treatment being the most effective (data not shown).

\section{Cell culture}

All cell lines were cultured under standard cell culture conditions $\left(37{ }^{\circ} \mathrm{C}, 5 \% \mathrm{CO}_{2}, 95 \%\right.$ rel. humidity). Information on the seeded cell densities and the used cell culture formats is summarized in the Supplementary Table 3. Cells were regularly checked for mycoplasma contamination.

H4IIE cells were incubated in MEM medium (PanBiotech $\mathrm{GmbH}$, Aidenbach, Germany) supplemented with $10 \% \mathrm{v} / \mathrm{v}$ fetal calf serum (Pan-Biotech $\mathrm{GmbH}$ ) and passaged every $2-3$ days after reaching an about $80 \%$ confluence. The treatment of the cells with the substances was performed in phenol-red-free MEM medium (Pan-Biotech $\mathrm{GmbH}$ ) supplemented with $10 \%$ v/v fetal calf serum (Pan-Biotech $\mathrm{GmbH}$ ).

HepG2 cells (ECACC, Porton Down, UK) were incubated in RPMI-1640 medium (Pan-Biotech $\mathrm{GmbH}$ ) supplemented with $10 \%$ v/v fetal calf serum (Pan-Biotech $\mathrm{GmbH}$ ). Cell passaging was conducted every 3-4 days after the cells reached a confluence of about $80 \%$. Treatment of the cells with the test substances was performed in phenol-red-free RPMI-1640 medium (Pan-Biotech GmbH) supplemented with $10 \%$ v/v fetal calf serum (Pan-Biotech $\mathrm{GmbH}$ ).

HepaRG cells were purchased from Biopredic International (Saint Grégoire, France) and were cultured as described by Lereau et al. (2012) with slight modifications. Briefly, the cells were expanded for 2 weeks in growth medium composed of William's E medium (Biochrom, Berlin, Germany) supplemented with $10 \% \mathrm{v} / \mathrm{v}$ fetal calf serum (Biochrom), $50 \mathrm{IU} / \mathrm{ml}$ penicillin $/ 50 \mu \mathrm{g} / \mathrm{ml}$ streptomycin (Biochrom), $2 \mathrm{mM} \mathrm{L-glutamine} \mathrm{(Biochrom),} 20 \mu \mathrm{g} / \mathrm{ml}$ gentamycin (Biochrom), $5 \mu \mathrm{g} / \mathrm{ml}$ human recombinant insulin (Biochrom) and $0.5 \mu \mathrm{g} / \mathrm{ml}$ dexamethasone (Sigma-Aldrich, Schnelldorf, Germany). For the subsequent differentiation period of 2 weeks and the further cultivation, the growth medium was additionally supplemented with $1.8 \%$ DMSO (Carl Roth) and $5 \mathrm{ng} / \mathrm{mL}$ human recombinant EGF (SigmaAldrich). Medium was changed every second day. Substance treatment followed 6 days after the end of the cell differentiation period.
Test concentrations were optimized for the different cell lines beforehand by evaluating the cytotoxicity (data not shown, the used nominal test concentrations are listed in the Supplementary Table 2). Only non-cytotoxic concentrations were used for the analyses. Cytotoxicity was monitored using the CytoTox-ONETM Homogeneous Membrane Integrity Assay (Promega, Madison, WI, USA), which checks for LDH leakage, and the WST-1 Cell Proliferation assay (Roche, Rotkreuz, Switzerland), which checks for mitochondrial metabolic activity of the cells. Both assays were performed according to the manufacturer's manual. In the modeling experiment, concentrations were adapted for modeling reasons.

\section{Molecular analysis}

RNA was isolated using peqGOLD TriFastTM (Peqlab, Erlangen, Germany) as recommended by the manufacturer. Subsequently, RNA was analyzed for quality and quantity with the Agilent Bioanalyzer (Agilent, Santa Clara, CA, USA).

\section{Whole genome microarray analysis}

The qualitatively best four out of six isolated RNA samples were used for the microarrays analyses. In detail, $200 \mathrm{ng}$ total RNA of all samples and the solvent-treated RNA control pool were prepared using the Low Input Quick Amp Labeling Kit (Agilent, Santa Clara, USA) for two-color microarray experiments. The following hybridization was conducted using the SurePrint platform (Agilent, Santa Clara, USA) and organism-specific designs of $8 \times 60 \mathrm{~K}$ microarray layouts from $R$. norvegicus (G3 Rat GE $8 \times 60 \mathrm{~K}, 30,003$ genes) and H. sapiens (G3 Human Gene Expression $8 \times 60 \mathrm{~K}$ v2, 50,599 genes). To monitor the experiments' accuracies, an external RNA was applied as control kit (RNA Spike-In Kit, Agilent, Santa Clara, USA). Washing of the slides was carried out using the Gene Expression Wash Buffer Kit (Agilent, Santa Clara, USA), and all signals were extracted and analyzed by the Agilent Feature Extraction software subsequent to the scanning process. Data were normalized using the Lowess method with EMMA2 software (Dondrup et al. 2009), and final signals were identified by performing a $t$ test with correction for multiple comparisons by adjusting the false discovery rate $(p<0.05$; Benjamini and Hochberg 1995). Based on a 'yellow' experiment, genes with $M$ values $>0.6$ and $<-0.6$ were defined as differentially expressed genes (DEGs). All M/A plots were generated by means of Microsoft Excel (2016), while Venn diagrams were generated by means of InteractiVenn (Heberle et al. 2015). Principal component analysis (PCA) was conducted by the usage of Omics Fusion (Brink et al. 2016). All results obtained from the human cell lines were analyzed for KEGG pathways by 
over-representation analysis (FDR $p<0.05$; Benjamini and Yekutieli 2001) with GeneTrail2 (Stoeckel et al. 2016).

\section{Quantitative real-time-PCR (qRT-PCR)}

The reverse transcription of $2 \mu \mathrm{g}$ RNA of the treated cells into cDNA was performed using the High Capacity cDNA Reverse Transcription Kit (Applied Biosystems, Darmstadt, Germany) according to the protocol of the manufacturer. Forty nanograms of cDNA was used for qRT-PCR analysis on an ABI 7900HT instrument (Applied Biosystems, Darmstadt, Germany) with Maxima SYBR Green/Rox qPCR Mastermix (Life Technologies, Carlsbad, USA) and $0.25 \mu \mathrm{M}$ primers (Eurofins, Luxemburg).

\section{Low-density qRT-PCR arrays}

For the low-density array analyses, $2 \mu \mathrm{g}$ RNA obtained from H4IIE cells after test substance incubation was reversely transcribed to cDNA using the RT ${ }^{2}$ First Strand cDNA Kit (Qiagen, Hilden, Germany) according to the protocol of the manufacturer. cDNA was analyzed by making use of the Molecular Toxicology PathwayFinder RT $^{2}$ ProfilerTM PCR Arrays (rat, \# PARN-3401ZE, SA Bioscience, Qiagen) on an ABI 7900HT real-time system (Applied Biosystems) as described by the manufacturer.

\section{Reporter gene assay}

HepG2 cells were used to establish a dual luciferase transactivation assay for the human CAR. In this assay, the first plasmid contains the sequence of a fusion protein of GAL4 with the ligand-binding domain of the human CAR and the second plasmid contains the firefly luciferase reporter gene under the control of five copies of the GAL4 upstream activation sequence (UAS) and a minimal thymidine kinase promoter. Cells were co-transfected with an expression vector for the human nuclear receptor hCAR. The third plasmid constitutively expresses the renilla luciferase sequence and serves as an internal control in all assays. HepG2 cells were transiently transfected using TransIT-LT1 (Mirus Bio LLC, Madison; WI, USA). After treatment and lysis of the cells (with $100 \mathrm{mM}$ potassium phosphate and $0.2 \%[\mathrm{v} / \mathrm{v}]$ Triton $\mathrm{X}-100, \mathrm{pH}$ 7.8), luminescence was measured using a plate reader (Infinite M200PRO, Tecan, Männedorf, Switzerland) according to the Dual Luciferase Assay protocol provided by the supplier (Promega).

\section{Analysis of the metabolome}

Treated cells and cell culture supernatants were harvested for metabolome analysis. About $7 \pm 1 \mathrm{mg}$ of the dried samples were homogenized using a Precellys 24 homogeniser (Bertin
Instruments, Frankfurt Main, Germany) at $3 \times 45 \mathrm{~s}, 6.5 \mathrm{~m} / \mathrm{s}$ with $0.2 \mathrm{~g}$ of silica beads and $1 \mathrm{ml} 80 \%$ methanol containing $10 \mu \mathrm{M}$ ribitol as internal standard. The exact dry weights of all samples were determined and used for later normalization. After centrifugation, $750 \mu \mathrm{l}$ of the supernatant was dried in a stream of nitrogen gas and transferred to the MultiPorpose Sampler (MPS2, Gerstel, Germany). The samples were subsequently derivatized with $100 \mu \mathrm{l}$ methoxyamine hydrochloride $(20 \mathrm{mg} / \mathrm{ml}$ in pyridine, Sigma-Aldrich) and $100 \mu \mathrm{l}$ MSTFA (Macherey-Nagel). Thereafter, they were injected for the analysis in a Leco Pegasus IV gas chromatograph (GC) coupled to time-of-flight mass spectrometer (MS; Leco, Saint Joseph, Michigan, USA). The GC instrument was equipped with an Rtx ${ }^{\circledR}-5 \mathrm{MS}$ column (30 m, iD $0.25, d f 0.25 \mu \mathrm{m}$; Restek). A 1- $\mu$ l sample was injected (splitless) for GC-MS analysis. The oven program was: $3 \mathrm{~min}$ $80{ }^{\circ} \mathrm{C}$, ramp with $5{ }^{\circ} \mathrm{C}$ per minute up to $325^{\circ} \mathrm{C}$, isothermic conditions for $2 \mathrm{~min}$ at $325^{\circ} \mathrm{C}$. The transfer line temperature was set at $250{ }^{\circ} \mathrm{C}$ and the ion source at $220^{\circ} \mathrm{C}$. Mass spectra were recorded from $\mathrm{m} / \mathrm{z} 50$ to 750 . The resulting chromatogram and mass spectra were converted to the Computable Data Format (CDF) and imported to the web-based software platform MeltDB 2.0 (Kessler et al. 2013) for further data analysis, statistical evaluation and visualization.

\section{Statistics and modeling}

SigmaPlot for Windows software (Version 14.0) and GraphPad Prism (Version 8.0) were used for statistical analysis and dose response modeling, respectively. The Shapiro-Wilks and Brown-Forsythe tests were applied to test for normal distribution and homogeneity of variances, respectively. Because most of the data did not meet the prerequisites for parametric testing, the non-parametric Mann-Whitney rank sum test was performed. Asterisks (*) indicate statistical significance at $p<0.05$, and error bars depict the standard deviation.

To obtain information on additivity of effects, a curve was modeled based on the data obtained for the individual substances by assuming concentration addition. The measured combination effect was then compared to the model, and a model deviation ratio (MDR) of two was accepted as a maximum deviation to accept additivity as suggested by Belden et al. (2007) and differentiate CA/DA from interaction (e.g., synergism or antagonism).

\section{Results}

Cells were treated with nominal concentrations of substances based on known in vivo effect concentrations and cytotoxicity testing (Table 1, Supplementary Table 2). Subsequent transcriptomics analysis revealed the number of 
Table 1 Measured concentrations in vivo

\begin{tabular}{|c|c|c|c|}
\hline \multirow[t]{2}{*}{ Substances } & \multicolumn{3}{|l|}{ In vivo } \\
\hline & $\begin{array}{l}\text { Nominal Concentration in } \\
\text { Diet }[\mathrm{mg} / \mathrm{kg}]\end{array}$ & $\begin{array}{l}\text { Measured concentration in liver } \\
( \pm \mathrm{SD})[\mathrm{mg} / \mathrm{kg}]\end{array}$ & $\begin{array}{l}\text { Measured concentration } \\
\text { in liver }[\mu \mathrm{M}]\end{array}$ \\
\hline Cyproconazole & 1000 & $15.6( \pm 1.52)$ & 53.5 \\
\hline Epoxiconazole & 900 & $8.2( \pm 3.26)$ & 24.9 \\
\hline Prochloraz & 1000 & $3.5( \pm 1.09)$ & 9.2 \\
\hline $\begin{array}{l}\text { Cyproconazole + Epoxiconazole } \\
\text { (Mixture I) }\end{array}$ & 1000 & $\begin{array}{l}5.2( \pm 2.13) \\
9.6( \pm 3.88)\end{array}$ & $\begin{array}{l}17.8 \\
29.1\end{array}$ \\
\hline $\begin{array}{l}\text { Cyproconazole }+ \text { Epoxiconazole }+ \\
\text { Prochloraz (Mixture II) }\end{array}$ & $\begin{array}{l}900 \\
1000\end{array}$ & $\begin{array}{l}8.4( \pm 5.55) \\
16.4( \pm 6.99) \\
1.6( \pm 1.59)\end{array}$ & $\begin{array}{l}29.0 \\
49.6 \\
4.2\end{array}$ \\
\hline
\end{tabular}

differentially expressed genes (DEGs), which was followed by a pathway-focused KEGG (Kyoto Encyclopedia of Genes and Genomes) analysis of transcriptomics data, a comparison of rat in vivo and rat in vitro data, and a comparison of rat in vitro data as well as human in vitro data. Based on the transcriptomics analysis, a common toxicity pathway and a corresponding biomarker were identified and applied for mixture toxicity modeling. Additionally, metabolomics analysis was used to support some of the findings on the affected toxicity pathways.

\section{The number of DEGs after a treatment with the single pesticides and their mixtures varies between the different cell lines}

The total number of DEGs in the case of the rat in vivo, rat in vitro (H4IIE) and human in vitro (HepaRG) is shown in Fig. 1a. Most genes were found to be deregulated in HepaRG cells (2058 genes just for the $\mathrm{P}$ treatment). In rat in vivo, the highest number of DEGs was observed in the CE-treated group (807 genes), and a maximal number of 381 genes was significantly deregulated in the H4IIE cell line by the CE mixture. However, when comparing both species, one has to take into account that the total number of genes analyzed in $R$. norvegicus is smaller than that in the human cell lines (30,003 vs 50,999 Entrez gene RNAs).

In a next step, we analyzed the overlap of individual DEGs by the different treatments, which was surprisingly small for C in HepaRG cells (Fig. 1a). Up to $79 \%$ (219 out of 278) of the DEGs in the HepaRG cells were altered by $\mathrm{C}$ only. However, depending on the substance or mixture, the overlap of individual DEGs became larger for $\mathrm{E}$ or $\mathrm{P}$.

Accordingly, only 13 genes were deregulated by all substance treatments in H4IIE cells (thereunder Gsta2, Ugt1a6, Ugt2b15 and Nqo1). This indicates that, despite the structural similarities of the substances, common biomarkers are difficult to identify when looking at the level of individual genes, with Cyp1a1 being one of the very few exceptions.

Additionally, the binary and ternary mixtures do not only deregulate the same genes stronger than the two triazole substances alone, but in addition they also deregulate some different genes (Fig. 1a). 37\% (142 out of 381) of the DEGs in H4IIE cells are additionally influenced by the CE mixture and again $37 \%$ (60 out of 164) by the CEP mixture when compared to the treatments with the single substances. In contrast, the structurally related but less similar azole fungicide $\mathrm{P}$ exclusively leads to $70 \%$ DEGs (236 out of 338).

In HepaRG cells, $62 \%$ (538 out of 862) of the DEG are influenced by CEP alone and not by the individual components of the mixture, while in the case of the CE mixture this level is much lower $(10 \%, 72$ of 784$)$. Here again, the azole fungicide $\mathrm{P}$ exclusively leads to $56 \%$ DEGs (1148 out of 2058).

The diagram also shows that the influence of epoxiconazole in H4IIE cells is higher than that of $\mathrm{C}$ in the $\mathrm{CE}$ mixture. The same also applies to the human cell line: The CE mixture effects on the transcriptome seem to be mainly driven by $\mathrm{E}$.

$\mathrm{P}$ seems to have the most distinct effect in all three cell lines, as a high number of genes are exclusively deregulated by $\mathrm{P}(70 \%, 236$ out of 338 in the H4IIE cells; $56 \%, 1148$ out of 2058 in the HepaRG cells), but not by any other treatment. This means that $\mathrm{P}$ with its distinct effects on the transcriptome mainly drives the effects in the mixtures containing $\mathrm{P}$, but shows a stronger impact when applied alone. The gene expression changes in the mixtures containing $\mathrm{P}$ seem to be antagonized by $\mathrm{C}$ and $\mathrm{E}$ in the H4IIE and HepaRG cells (Fig. 1a).

\section{Overlap of DEGs is low in the case of the three fungicides and the three different models used}

Figure $1 \mathrm{~b}$ compares the overlap of DEGs by treatment with the single substances $\mathrm{P}, \mathrm{C}$ and $\mathrm{E}$ in the three models 


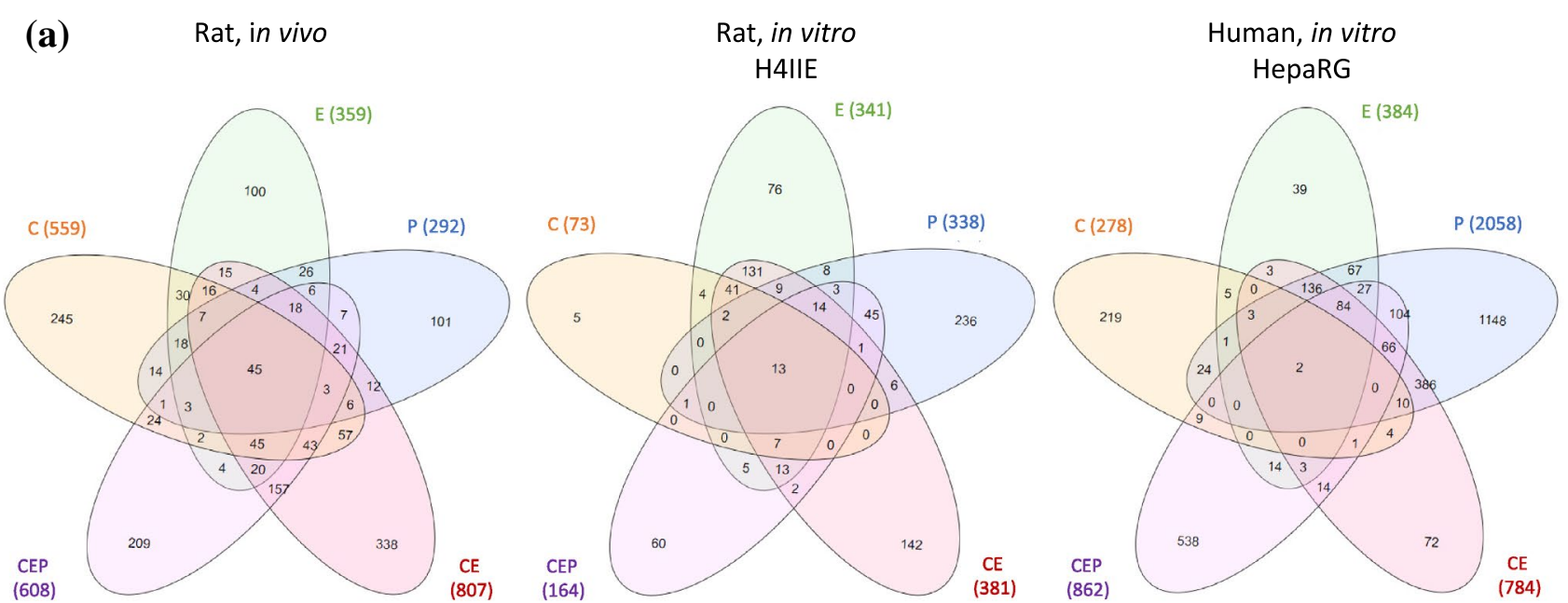

(b)
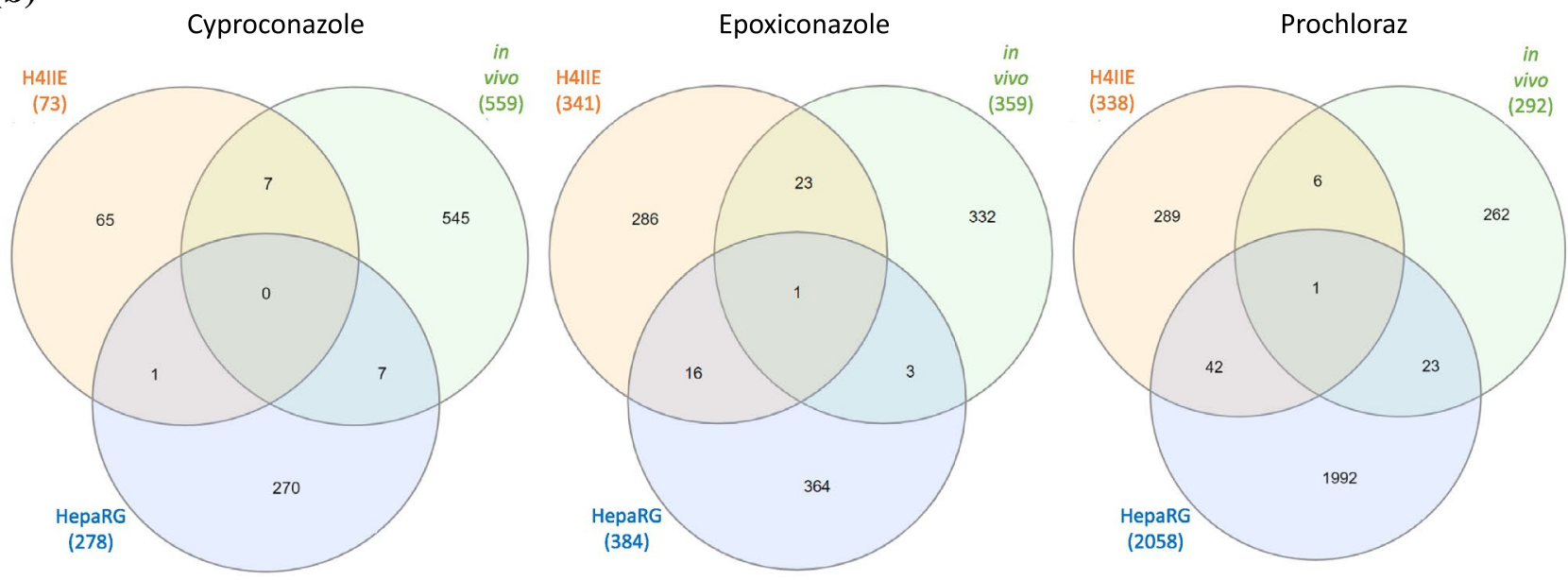

Fig. 1 Venn diagrams of DEGs in comparison to the solvent control $(M>=0.6, M<=-0.6, p<0.01)$ and comparison of in vivo rat with the rat cell line H4IIE and the human cell line HepaRG under different treatments: cyproconazole $(\mathrm{C})$, epoxiconazole $(\mathrm{E})$, prochloraz $(\mathrm{P})$, cyproconazole + epoxiconazole (CE) and cyproconazole + epoxicona- zole + prochloraz (CEP). a Sorted by test system, comparing the treatments; b sorted by treatment, comparing the test systems. Binary and ternary mixtures of test substances were applied in equimolar mixtures (e.g., $2.5 \mu \mathrm{M}$ cyproconazole $+2.5 \mu \mathrm{M}$ epoxiconazole). Nominal test substance concentrations are listed in Supplementary Table 2 (rat in vivo, rat in vitro H4IIE and human in vitro HepaRG). The overlap is low taking into account that only one gene is commonly affected in all three models by $\mathrm{P}$ (Ephx1). In the case of $\mathrm{P}$, the overlap between the two cell lines is higher than the overlap between rat in vivo and in vitro, which potentially points out to different toxicokinetics or metabolism in vivo and may in turn result in a different set of DEGs.

The overlap of all three models is even lower for C (no DEG). However, here the overlap between rat in vivo and in vitro is higher than the overlap between H4IIE and HepaRG cells, thereby pointing out to species differences in reaction to this substance.
Transcriptomic data from H4IIE cells treated with the mixtures and confirmed by qRT-PCR show a good correlation with data from an in vivo study in rats

Table 2 shows the gene expression data for selected target genes from the microarray and RT-qPCR analyses in the H4IIE cells in comparison to data from the low-density arrays in rat livers obtained from a previously performed 28-day feeding study (Heise et al. 2018) for C, E and CE. The data from the microarray, the low-density array and the RT-qPCR analyses obtained in the in vitro experiments and the in vivo study show a good correlation. Cumulative 
effects were obvious for Cyp1a1 induction in vitro and in vivo.

To further confirm the microarray data, qRT-PCR analyses were conducted for selected deregulated top scorer genes from microarray analyses to demonstrate concentrationdependent effects. The Cyp1a1 expression in the human HepG2 and the rat H4IIE cells after an incubation with increasing concentrations of cyproconazole, epoxiconazole and its mixture is exemplarily shown in the Supplementary Fig. 1. Data from the microarray analyses were additionally confirmed using a low-density array for a broad range of genes in the H4IIE cell line treated with C, E, P, CE and CEP (Supplementary Table 4). A high number of genes were deregulated by the (tri)azole treatment, and the substance combinations seem to have cumulative effects affecting a number of genes (e.g., Aass, Abcc2, Ahr, Cyp1a1, Gadd45a and Mgmt). The most pronounced effects were caused by the treatment with the ternary mixture CEP and the single substance prochloraz.

\section{Pathway analysis reveals a high level of similarity between rat in vivo, rat in vitro and human cell lines, but not for all substances}

A KEGG pathway analysis of the DEGs in rat in vivo as well as in the treated H4IIE and HepaRG cells was performed, and the most strongly affected KEGG pathways are shown in Fig. 2 and Table 3. In contrast to the observation made in the case of individual DEGs, where the overlap was quite low, the level of similarity became higher, when genes were assigned to toxicity pathways.

In rat liver in vivo, the five most affected KEGG pathways were essentially similar: chemical carcinogenesis, xenobiotic metabolism, drug metabolism, steroid hormone metabolism and retinol metabolism (see Fig. 2) were most affected by all three substances as well as by the combinations.

In the rat liver cell line H4IIE, the five most affected KEGG pathways were chemical carcinogenesis, xenobiotic metabolism, drug metabolism, steroid hormone metabolism and retinol metabolism in the case of $\mathrm{C}$, chemical carcinogenesis, xenobiotic metabolism, drug metabolism and retinol metabolism followed by pentose and glucuronate metabolism instead of steroid metabolism in the case of epoxiconazole and chemical carcinogenesis, xenobiotic metabolism, drug metabolism, pentose and glucuronate metabolism and bile secretion in the case of prochloraz. In the case of the mixtures, xenobiotic metabolism, drug metabolism, chemical carcinogenesis, pentose and glucuronate metabolism and retinol metabolism were most affected. Overall, these effects show a good correlation with the situation in vivo, with the three most affected pathways being similar, while some differences regarding certain pathways are evident (Fig. 2). 

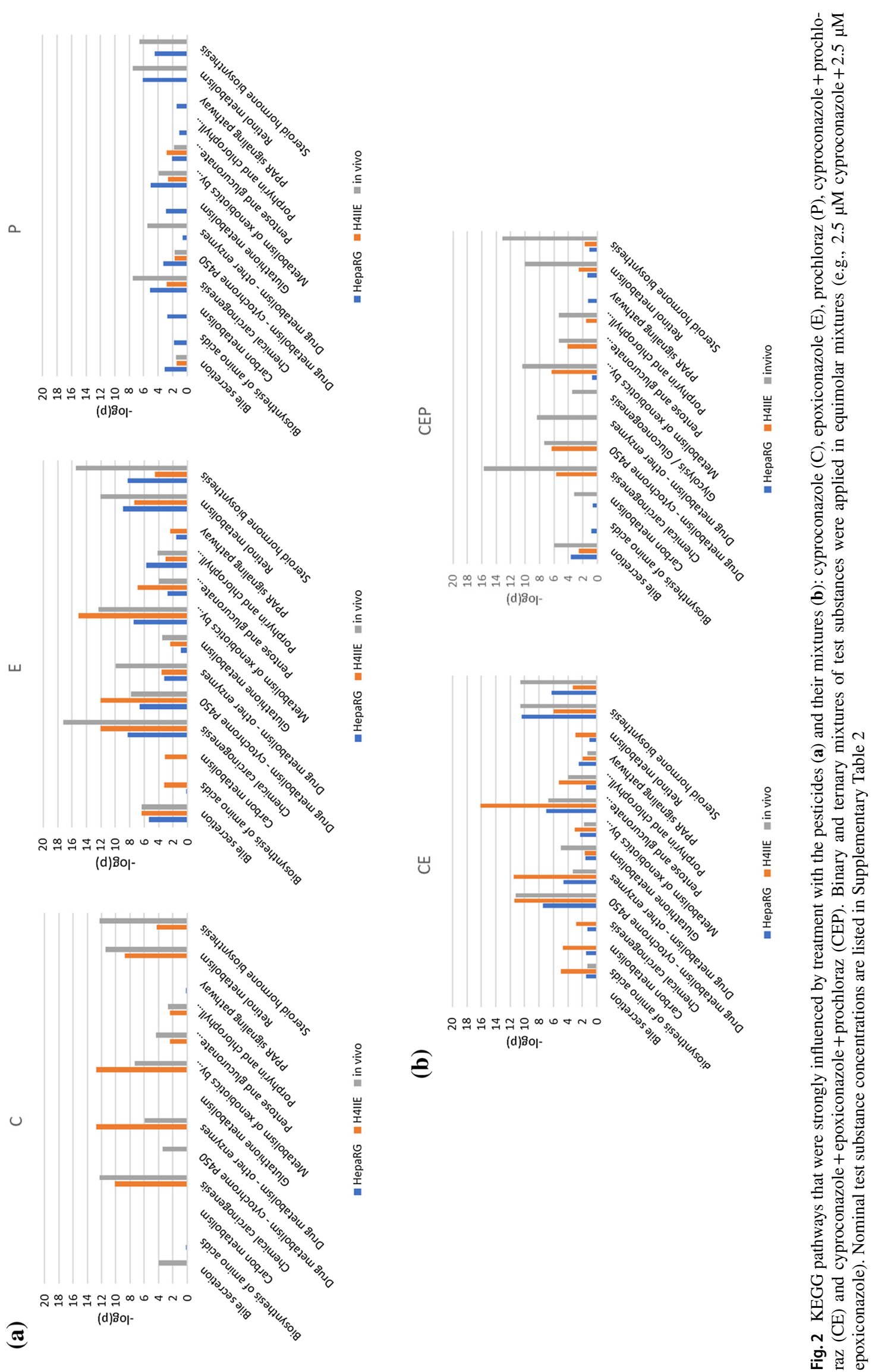
Table 3 Amount of differentially expressed genes (DEG) sorted by KEGG pathway. The sum of DEG affected in vivo in a rat 28-day study and in vitro in H4IIE cells and HepaRG cells is shown

\begin{tabular}{|c|c|c|c|}
\hline KEGG pathway & $\sum$ in vivo & $\sum$ H4IIE & $\sum$ HepaRG \\
\hline Bile secretion & 19.1 & 15.4 & 13.6 \\
\hline Biosynthesis of amino acids & 0.0 & 7.9 & 4.5 \\
\hline Carbon metabolism & 3.2 & 6.0 & 4.7 \\
\hline Chemical carcinogenesis & 63.9 & 42.1 & 20.8 \\
\hline Drug metabolism—cytochrome P450 & 23.8 & 31.6 & 14.5 \\
\hline Drug metabolism—other enzymes & 34.8 & 18.0 & 5.4 \\
\hline Glutathione metabolism & 8.7 & 5.5 & 6.1 \\
\hline Metabolism of xenobiotics by cytochrome $\mathrm{P} 450$ & 40.8 & 53.0 & 20.2 \\
\hline Pentose and glucuronate interconversions & 19.4 & 21.7 & 6.4 \\
\hline Porphyrin and chlorophyll metabolism & 13.5 & 8.9 & 9.3 \\
\hline PPAR signaling pathway & 0.0 & 5.4 & 5.5 \\
\hline Retinol metabolism & 51.6 & 24.7 & 26.9 \\
\hline Steroid hormone biosynthesis & 58.1 & 13.9 & 20.2 \\
\hline
\end{tabular}

In the human liver cell line HepaRG, the only affected pathways observed after treatment with cyproconazole were the PPAR signaling pathway and amino acid metabolism. This differed strongly from $\mathrm{E}, \mathrm{P}$ and the binary combination, in which case the five most affected pathways were chemical carcinogenesis, xenobiotic metabolism, drug metabolism, steroid hormone metabolism and retinol metabolism. This may partially be explained by species differences observed for cyproconazole in terms of the substances' ability to activate the human forms of the nuclear receptors CAR and PXR (e.g., Marx-Stoelting et al. 2017), both considered to play a central role in the induction of hepatotoxicity by this fungicide in rats.

While in the case of the rat liver in vivo and in vitro the level of similarity for all three substances and the mixtures can be regarded as high, there are clear differences regarding the pathways affected in the H4IIE cells (rat hepatic origin) and the HepaRG cells (human hepatic origin).

Again, it is evident, as already seen when comparing DEGs in the different cell lines (Fig. 1a), that the treatment with $\mathrm{C}, \mathrm{E}$ and $\mathrm{CE}$ shows a higher concordance regarding the DEGs and affected KEGG pathways if compared to $\mathrm{P}$ and mixtures containing $\mathrm{P}$. This may again point at a different mode of action of this compound.

\section{Information on the affected toxicity pathways can be used to select potential biomarkers for mixture toxicity assessment}

Pathway-focused analysis revealed that toxicity pathways affecting drug and xenobiotic metabolism are the most commonly affected pathways by all substances. Potential molecular initiating events of these pathways consist of activation of nuclear receptors such as AhR and/or CAR. Since the ability of the substances used in this study to activate AhR has already been analyzed elsewhere (e.g., Rieke et al. 2014), we focused on CAR transactivation. Figure $3 \mathrm{a}$ shows that CAR is indeed activated by cyproconazole and epoxiconazole. The reporter gene assay was also used to test if mixture effects observed are in line with DA/CA model. As demonstrated in Fig. 3a, the effects observed for C, E and the binary mixture are in line with the dose/concentration addition model.

The only marker gene that was altered throughout treatments and models was CYP1A1. Hence, we used CYP1A1 for a similar modeling approach (Fig. 3b). In the case of CYP1A1 expression, concentration addition was also observed.

\section{The treatment with different pesticides and their mixtures leads to distinct metabolome profiles}

In addition to the transcriptome analysis, the metabolome of H4IIE cells and their supernatants were analyzed. The metabolome profile after treatment with cyproconazole, epoxiconazole and their binary mixture in a PCA is shown in Supplementary Fig. 2. The samples can be clearly segregated and the treated cells can be distinguished from the solvent control when analyzing the cells as well as the cell culture supernatants. The PCA, besides the cluster analysis, demonstrates that the combination of the two pesticides (CE) induces the most significant differences, followed by the treatment with $\mathrm{E}$ alone, which is comparable to the transcriptomic profile. The mixture effects of the CE treatment seem to be primarily determined by the influence of E, which is in line with the transcriptomics analyses. A particular difficulty of the metabolome analysis is the definitive identification of the metabolites. Sixty identified metabolites are demonstrated in Supplementary Table 5. As most of the identified metabolites are components of the central metabolism and particularly sugars, intermediate products of glycolysis or amino 
(a)

\section{CAR-Dual-Luciferase-Assay in HepG2}

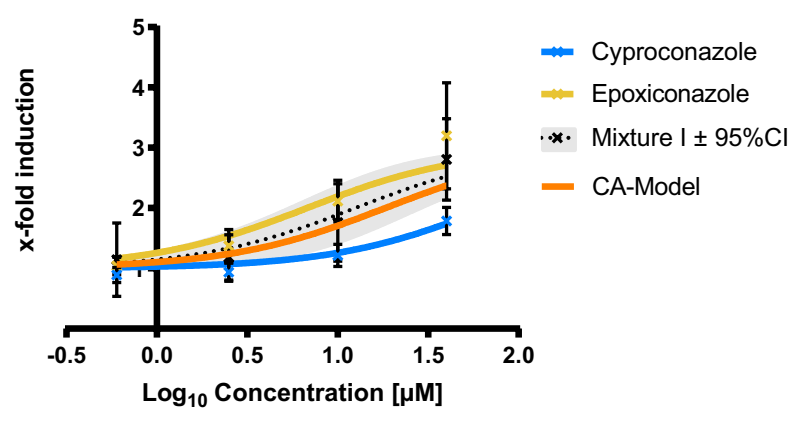

(b)

\section{CYP1A1-Expression in HepaRG}

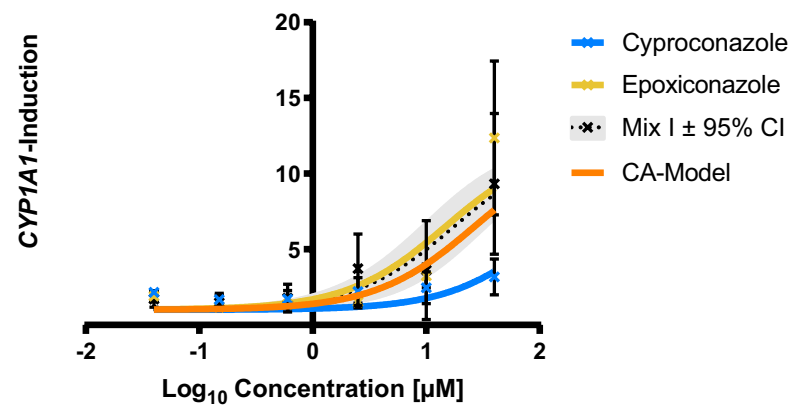

Fig. 3 Biomarkers obtained by the omics analysis were used for a more comprehensive mixture toxicity analysis and modeling of concentration-response curves to compare the measured curves with those obtained by modeling by use of the concentration addition model. a As found out by omics-based mode of action analysis, CAR is important for the MOA of $\mathrm{C}$ and $\mathrm{E}$. Results of a reporter gene assay in HepG2 cells are presented. b Results for Cyp1a1, the only comprehensive biomarker gene found: Results are in line with the concentration addition model. Please note that for modeling reasons, the total azole concentration is presented on the $\mathrm{x}$-axis (e.g., at $5 \mu \mathrm{M}$, individual substances have a concentration of $5 \mu \mathrm{M}$, while the total azole concentration in the mixture is also $5 \mu \mathrm{M}$, consisting of $2.5 \mu \mathrm{M} \mathrm{C}$ and $2.5 \mu \mathrm{M} \mathrm{E}$ in the binary mixture)

acids, it was difficult to correlate the data of particular metabolic pathways to the transcriptomics findings. However, approximately half of the metabolites identified could be assigned to either 'Pentose and gluconate', 'steroid hormone biosynthesis' or 'biosynthesis of amino acids'; three pathways were found to be strongly altered according to the KEGG analysis of the transcriptomics data.

\section{Discussion}

The aim of the present study was to analyze combination effects of three azole fungicides in vitro by use of omics techniques and, by doing so, to identify potential biomarkers of mixture toxicity and mode of action for further testing and modeling. Moreover, the study should shed light on the added value of omics techniques in the risk assessment of fungicide mixtures and the development of strategies for mixture toxicity testing.

\section{Omics data help to identify similarities in modes of action}

At a first glance, the congruence regarding the genes affected by each of the three fungicides in rats and rat liver cells on the one hand and hepatic cells of human origin on the other hand seems to be rather disappointing. When looking at the number of genes affected, only a very few genes remain, among them Cyp1a1, which has previously been described as a potential marker in other test systems (Rieke et al. 2014). Considering the low level of concordance when looking at the level of individual genes, the results seem to be less surprising. For example, Ochsner et al. (2009) showed that omics analysis of the same experiment (treatment of MCF-7 cells with E2) under exactly the same conditions and performing the same microarray experiment multiple times results in a very limited overlap of common genes identified. This changes, when a pathway-focused analysis is performed instead of looking at similarity at the level of individual genes. In our analysis, five pathways were identified as being affected in rats and humans in vitro and in vivo by the two triazoles $\mathrm{C}$ and $\mathrm{E}$ and their mixture: chemical carcinogenesis, xenobiotic metabolism, drug metabolism, retinol metabolism and steroid metabolism. This analysis is considerably well in line with results by others for the triazole fungicides myclobutanil, propiconazole and triadimefon in rats (Goetz and Dix 2009a, b). A key receptor in activating these pathways is CAR, which is considered to be an MIE in adverse outcome pathways for chemical hepatocarcinogenesis and a key mediator of triazole toxicity (Goetz and Dix 2009a; Marx-Stoelting et al. 2017).

The involvement of retinoic acid signaling in triazolemediated developmental toxicity has previously been described based on transcriptomics results from rat whole embryo cultures treated with cyproconazole, flusilazole or triadimefon (Robinson et al. 2012) and our results are in line with these findings.

Only prochloraz seems to trigger additional toxicity pathways. This is also in line with previous observations by our research group and others, showing that $\mathrm{P}$ is an activator of AhR (e.g., Rieke et al. 2014). Since AhR-mediated signals are considered to counteract to some extent CARmediated signals (Braeuning et al. 2009), it is not surprising that the number of affected genes declines when the AhR activator prochloraz is applied in combination with the CAR activators $\mathrm{C}$ and $\mathrm{E}$. 


\section{Species specificity of triazole-mediated hepatotoxicity}

At a first glance, the overlap of genes affected by each of the three fungicides in rats and rat liver cells on the one hand and hepatic cells of human origin on the other hand is very limited. While some similarities are evident, there is no overlap at all for $\mathrm{C}$ when rat and human gene sets are compared. However, species differences have previously been observed for $\mathrm{C}$, e.g., in mice bearing the human form of the receptors CAR and PXR, in which case a weak activation (if at all) was observed (Marx-Stoelting et al. 2017). This observation is in line with our findings and in support of the models compared in the present study. In fact, it illustrates the need to take into account species differences in addition to mode of action, when combination effects are to be addressed. In contrast, at the pathway level, a conservation has previously been observed for triadimefon, propiconazole and myclobutanil (Goetz and Dix 2009b).

\section{Relevance of omics data for risk assessment of pesticide mixtures}

The usage of receptor-based reporter assays has been established for the quantitative assessment of chemical mixture effects, especially for substances binding to hormone receptors, by means of concentration addition for substances sharing a mode of action (MoA) or independent action for substances with dissimilar MoA (Orton et al. 2014; Seeger et al. 2016). This system has also been used in the present study and was again capable of showing that the substances used follow the DA/CA model. However, the applicability of complex omics data in risk assessment is currently quite limited (Marx-Stoelting et al. 2015). Thus, omics technologies are primarily used as additional information for the identification of important MoA and they seem to be most promising in the framework of an integrated test strategy for the identification of biomarkers for adverse effects (Buesen et al. 2017). On the one hand, omics data are considered to be useful for mode of action analysis or prioritization of

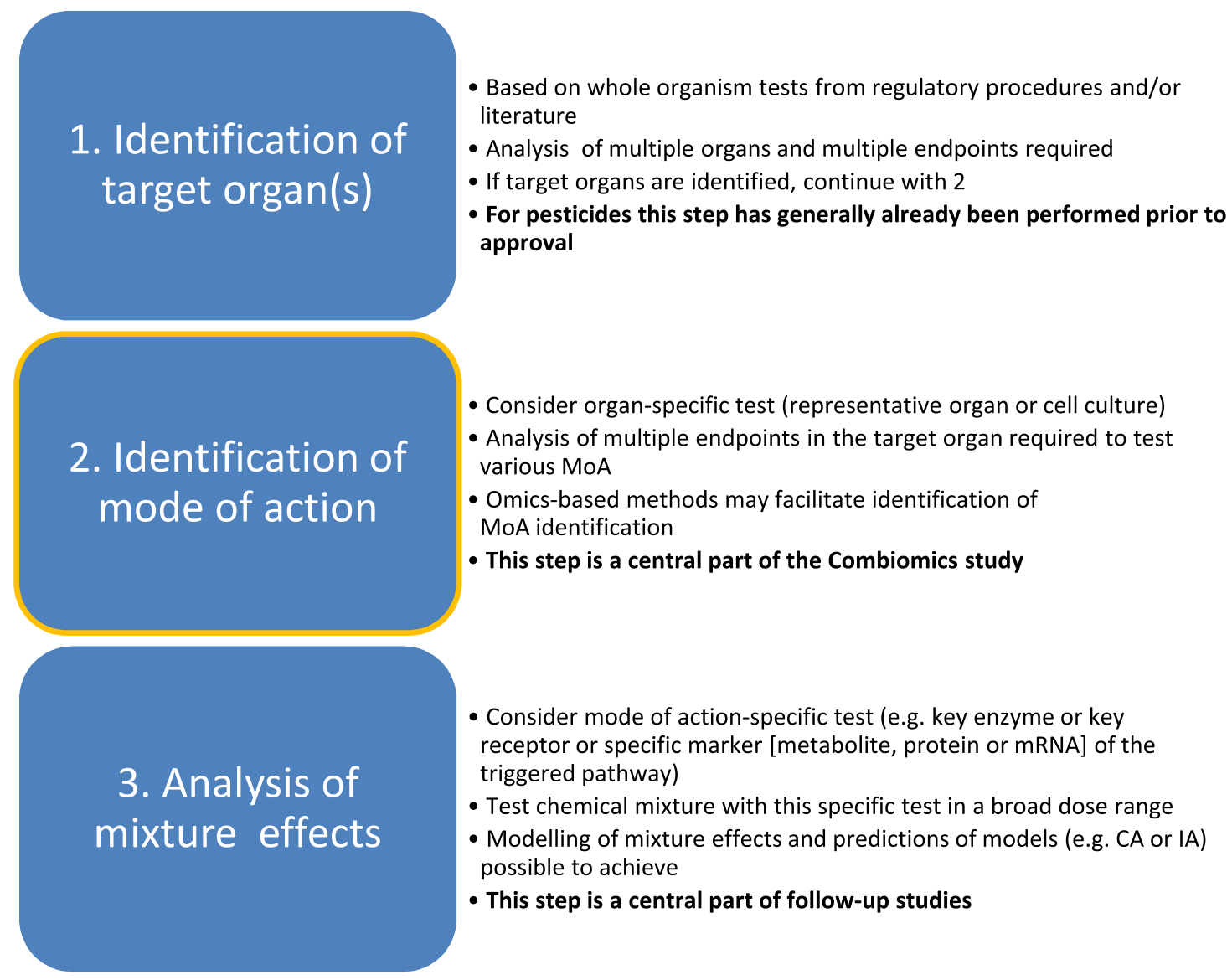

Fig. 4 The Combiomics testing strategy. Stepwise procedure for mixture toxicity testing and assessment based on target organ identification, omics-based mode of action/adverse outcome pathway analysis and detailed mixture analysis and modeling based on key pathways affected. The central step of the Combiomics project (this study) is marked in orange 
substances for further testing; on the other hand, the use of such data for purposes such as deriving reference values is not generally supported (e.g., Marx-Stoelting et al. 2015; Brockmeier et al. 2017). The identification of common modes of action of pesticides helps to group substances into cumulative assessment groups (CAGs), which is also possible with the help of in vitro assays. Thus, as a result of this study, we can conclude that a testing strategy for mixture toxicity requires the inclusion of omics techniques for MOA analysis rather than being based on them.

\section{Toward a strategy for mixture toxicity testing}

A central aim of the project was to contribute to the development of a strategy for mixture toxicity testing. Key steps of such a strategy for data-rich substances like pesticide actives are presented in Fig. 4. The major regulatory question is whether substances in a mixture perform according to the regulatory default assumption, which is dose or concentration addition, or if they perform less well (Solecki et al. 2014). Thus, a testing strategy would not necessarily have to ultimately clarify but would have to prioritize mixtures of concern for further testing based on mechanistic information. Hence, a mechanistic analysis is important to identify MOA and/or AOP affected by the substances, followed then by modeling of mixture toxicity by the use of common biomarkers to clarify if the DA/CA model is met or not. This objective can clearly be reached with the approach presented in this study.

Funding This study was funded by the German Federal Ministry of Education and Research, BMBF (e:TOP), Grant 031A267A, 031A267B, 031A267C.

\section{Compliance with ethical standards}

Conflict of interest The authors declare to have no conflict of interest.

Open Access This article is distributed under the terms of the Creative Commons Attribution 4.0 International License (http://creativeco mmons.org/licenses/by/4.0/), which permits unrestricted use, distribution, and reproduction in any medium, provided you give appropriate credit to the original author(s) and the source, provide a link to the Creative Commons license, and indicate if changes were made.

\section{References}

Altenburger R, Scholz S, Schmitt-Jansen M, Busch W, Escher BI (2012) Mixture toxicity revisited from a toxicogenomic perspective. Environ Sci Technol 46(5):2508-2522. https://doi. org/10.1021/es2038036

Ankley GT, Bennett RS, Erickson RJ et al (2010) Adverse outcome pathways: a conceptual framework to support ecotoxicology research and risk assessment. Environ Toxicol Chem 29(3):730741. https://doi.org/10.1002/etc.34

Belden JB, Gilliom RJ, Lydy MJ (2007) How well can we predict the toxicity of pesticide mixtures to aquatic life? Integr Environ Assess Manag 3(3):364-372

Benjamini Y, Hochberg Y (1995) Controlling the false discovery rate: a practical and powerful approach to multiple testing. J R Stat Soc: Ser B (Methodol) 57(1):289-300

Benjamini Y, Yekutieli D (2001) The control of the false discovery rate in multiple testing under dependency. Ann Stat 29(4):1165-1188

Braeuning A, Sanna R, Huelsken J, Schwarz M (2009) Inducibility of drug-metabolizing enzymes by xenobiotics in mice with liver-specific knockout of Ctnnb1. Drug Metab Dispos 37(5):1138-1145

Brink BG, Seidel A, Kleinbolting N, Nattkemper TW, Albaum SP (2016) Omics fusion-a platform for integrative analysis of omics data. J Integr Bioinform 13(4):296. https://doi. org/10.2390/biecoll-jib-2016-296

Brockmeier EK, Hodges G, Hutchinson TH, Butler E, Hecker M, Tollefsen KE, Garcia-Reyero N, Kille P, Becker D, Chipman K, Colbourne J, Collette TW, Cossins A, Cronin M, Graystock P, Gutsell S, Knapen D, Katsiadaki I, Lange A, Marshall S, Owen SF, Perkins EJ, Plaistow S, Schroeder A, Taylor D, Viant M, Ankley G, Falciani F (2017) The role of omics in the application of adverse outcome pathways for chemical risk assessment. Toxicol Sci 158(2):252-262. https://doi.org/10.1093/toxsci/ kfx097

Buesen R, Chorley BN, da Silva Lima B et al (2017) Applying 'omics technologies in chemicals risk assessment: report of an ECETOC workshop. Regul Toxicol Pharmacol. https://doi.org/10.1016/j. yrtph.2017.09.002

Dondrup M, Albaum SP, Griebel T et al (2009) EMMA 2-a MAGEcompliant system for the collaborative analysis and integration of microarray data. BMC Bioinform 10:50. https://doi. org/10.1186/1471-2105-10-50

EC (2009) Regulation (EC) No 1107/2009 of the European Parliament and of the Council of 21 October 2009 Concerning the Placing of Plant Protection Products on the Market and Repealing Council Directives 79/117/EEC and 91/414/EEC

EFSA (2008) Conclusion on the peer review of epoxiconazole. EFSA Sci Rep 138:1-80

EFSA (2010) Conclusion on the peer review of the risk assessment of the active substance cyproconazole. EFSA J 8(11):1897

EFSA (2011) Conclusion on the peer review of the pesticide risk assessment of the active substance prochloraz. EFSA J 9(7):2323

EFSA (2018) The 2016 European Union report on pesticide residues in food. EFSA J 16(7):5348. https://doi.org/10.2903/j.efsa.2018.5348

EFSA PPR Panel (2009) Risk Assessment for a selected group of pesticides from the triazole group to test possible methodologies to assess cumulative effects from exposure throughout food from these pesticides on human health. EFSA J 7(9):1167, Available online: https://www.efsa.europa.eu/de/efsajournal/pub/1167. Last Accessed 07 Nov 2017

EFSA Scientific Committee (2019) Guidance on harmonised methodologies for human health, animal health and ecological risk assessment of combined exposure to multiple chemicals. EFSA J 17(3):5634. https://doi.org/10.2903/j.efsa.2019.5634

Goetz AK, Dix DJ (2009a) Mode of action for reproductive and hepatic toxicity inferred from a genomic study of triazole antifungals. Toxicol Sci 110(2):449-462. https://doi.org/10.1093/toxsci/kfp098

Goetz AK, Dix DJ (2009b) Toxicogenomic effects common to triazole antifungals and conserved between rats and humans. Toxicol Appl Pharmacol 238(1):80-89. https://doi.org/10.1016/j. taap.2009.04.016

Heberle H, Meirelles GV, da Silva FR, Telles GP, Minghim R (2015) InteractiVenn: a web-based tool for the analysis of sets through 
Venn diagrams. BMC Bioinform 16:169. https://doi.org/10.1186/ s12859-015-0611-3

Heise T, Schmidt F, Knebel C et al (2015) Hepatotoxic effects of (tri)azole fungicides in a broad dose range. Arch Toxicol 89(11):2105-2117. https://doi.org/10.1007/s00204-014-1336-1

Heise T, Schmidt F, Knebel C et al (2018) Hepatotoxic combination effects of three azole fungicides in a broad dose range. Arch Toxicol. https://doi.org/10.1007/s00204-017-2087-6

Kessler N, Neuweger H, Bonte A et al (2013) MeltDB 2.0-advances of the metabolomics software system. Bioinformatics 29(19):24522459. https://doi.org/10.1093/bioinformatics/btt414

Kortenkamp A, Backhaus T, Faust M (2009) State of the art report on mixture toxicity

Lereau M, Gouas D, Villar S et al (2012) Interactions between hepatitis $B$ virus and aflatoxin $B(1)$ : effects on $p 53$ induction in HepaRG cells. J Gen Virol 93(Pt 3):640-650. https://doi.org/10.1099/ vir.0.032482-0

Marx-Stoelting P, Braeuning A, Buhrke T et al (2015) Application of omics data in regulatory toxicology: report of an international BfR expert workshop. Arch Toxicol 89(11):2177-2184. https:// doi.org/10.1007/s00204-015-1602-x

Marx-Stoelting P, Ganzenberg K, Knebel C et al (2017) Hepatotoxic effects of cyproconazole and prochloraz in wild-type and hCAR/hPXR mice. Arch Toxicol 91(8):2895-2907. https://doi. org/10.1007/s00204-016-1925-2

Ochsner SA, Steffen DL, Hilsenbeck SG, Chen ES, Watkins C, McKenna NJ (2009) GEMS (Gene Expression MetaSignatures), a Web resource for querying meta-analysis of expression microarray datasets: 17beta-estradiol in MCF-7 cells. Cancer Res 69(1):2326. https://doi.org/10.1158/0008-5472.CAN-08-3492

OECD (2018) Considerations for assessing the risks of combined exposure to multiple chemicals. OECD Series on Testing and Assessment No 296

Orton F, Ermler S, Kugathas S, Rosivatz E, Scholze M, Kortenkamp A (2014) Mixture effects at very low doses with combinations of anti-androgenic pesticides, antioxidants, industrial pollutant and chemicals used in personal care products. Toxicol Appl Pharmacol 278(3):201-208. https://doi.org/10.1016/j.taap.2013.09.008

Rieke S, Koehn S, Hirsch-Ernst K, Pfeil R, Kneuer C, Marx-Stoelting $\mathrm{P}$ (2014) Combination effects of (tri)azole fungicides on hormone production and xenobiotic metabolism in a human placental cell line. Int J Environ Res Public Health 11(9):9660-9679. https:// doi.org/10.3390/ijerph110909660

Robinson JF, Tonk EC, Verhoef A, Piersma AH (2012) Triazole induced concentration-related gene signatures in rat whole embryo culture. Reprod Toxicol 34(2):275-283. https://doi.org/10.1016/j. reprotox.2012.05.088

Schmidt F, Marx-Stoelting P, Haider W et al (2016) Combination effects of azole fungicides in male rats in a broad dose range. Toxicology 355-356:54-63. https://doi.org/10.1016/j.tox.2016.05.018

Seeger B, Klawonn F, Nguema Bekale B, Steinberg P (2016) Mixture effects of estrogenic pesticides at the human estrogen receptor $\alpha$ and $\beta$. PLoS One 11(1):e0147490. https://doi.org/10.1371/journ al.pone. 0147490

Solecki R, Stein B, Frische T, Matezki S, Wogram J, Streloke M (2014) Paradigm shift in the risk assessment of cumulative effects of pesticide mixtures and multiple residues to humans and wildlife: German proposal for a new approach. J Consum Prot Food Saf 9(4):329-331. https://doi.org/10.1007/s00003-014-0914-8

Stoeckel D, Kehl T, Trampert P et al (2016) Multi-omics enrichment analysis using the GeneTrail2 web service. Bioinformatics 32(10):1502-1508. https://doi.org/10.1093/bioinformatics/btv770

Vinken M (2013) The adverse outcome pathway concept: a pragmatic tool in toxicology. Toxicology 312:158-165. https://doi. org/10.1016/j.tox.2013.08.011

Publisher's Note Springer Nature remains neutral with regard to jurisdictional claims in published maps and institutional affiliations. 